\title{
RESEÑA DE LIBRO: VIOLENCIA \\ EN EL FÚTBOL. INVESTIGACIONES SOCIALES Y FRACASOS POLÍTICOS
}

\author{
RESENHA DO LIVRO: VIOLÊNCIA NO FUTEBOL. \\ INVESTIGAÇÃO SOCIAL E FRACASSOS POLÍTICOS \\ BOOK REVIEW: SOCCER VIOLENCE SOCIAL RESEARCHES \\ AND POLITIC FAILURES
}

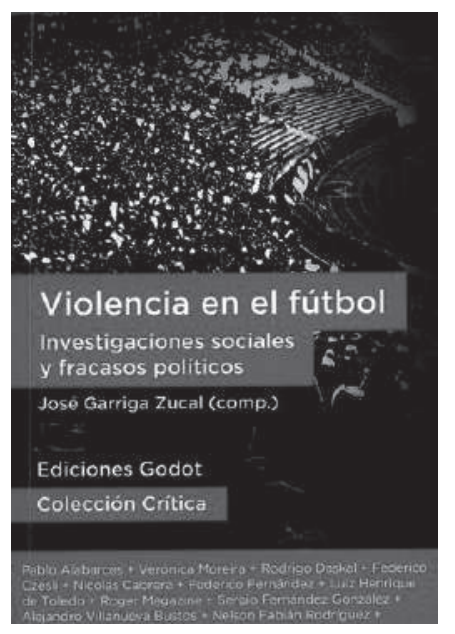

Título de la obra: Violencia en el fútbol.

Investigaciones sociales y fracasos políticos.

Autor. José Garriga Zucal (Compilador)

Año de edición: 2013

Editorial: Ediciones Godot

Lugar de edicicón: Buenos Aires, Argentina.

ISBN: 978-987-1489-67-1

Número de Páginas: 413

Castro, L. J. A. (2015). Rese a de libro: Violencia en el f tbol. Investigaciones sociales y fracasos pol ticos. Educaci n F sica y Deporte, 34 (2), 583-591 Jul.-Dic. http://doi. org/10.17533/udea.efyd.v34n2a13 


\section{JOHN ALEXANDER CASTRO LOZANO}

Soci logo de la Universidad Nacional de Colombia y Mag ster en Estudios Sociales de la Universidad Pedag gica Nacional (Colombia).

Profesor de sociolog a en la Universidad Antonio Nari o (Bogot - Colombia) jacastrol@uan.edu.co 
La violencia en el $f$ tbol es una afirmaci $n$ que debe contextualizarse ya que su significado es m Itiple, al depender de qui $n$ la define, desde qu perspectiva, qu posici $\mathrm{n}$ tiene y en qu momento lo hace. As, el libro compilado por Jos Garriga contribuye a la investigaci n sobre las hinchadas, barras bravas o los grupos organizados de hinchas pues examina las pr cticas, las representaciones y los sentidos que elaboran los hinchas, respecto a la violencia o el aguante. Asimismo, enfatiza que la pol tica $p$ blica se ha ocupado de castigar a los "violentos". Pero no ha analizado las causas que producen esas violencias pues no es esa su finalidad. En otras palabras, de lo que se trata es comprender los comportamientos de los grupos organizados de hinchas, con el prop sito de elaborar una pol tica p blica, que afronte este fen meno urbano y no sea, necesariamente, represiva.

Violencia en el $f$ tbol compila catorce trabajos de investigaci $n$, que incluye a especialistas de Argentina, Brasil, Colombia y $M$ xico. El libro se organiza en tres secciones. En la primera parte, Violencias locales: abordajes, miradas y diagn sticos, se encuentran los documentos de Pablo Alabarces, Ver nica Moreira, Rodrigo Daskal, Federico Czesli y Nicol s Cabrera. En la segunda parte, Otras tramas, otros problemas y algunas soluciones, son presentados los documentos de Federico Fern ndez, Roger Magazine, Sergio Fern ndez, Luiz Henrique De Toledo, Alejandro Villanueva, Nelson Rodr guez, Fernando Segura y Diego Murzi. Y en la tercera parte, La gesti $n$ de la seguridad, se re nen los textos de Santiago Uliana, Mat as Godio, Juan Sodo, Sebasti n Sustas y Jos Garriga. En la introducci n, "Cartograf a de la(s) violencia(s)", Garriga advierte que el concepto de violencia tiene varios significados pues es una manifestaci n construida social e hist ricamente. Si bien la violencia en el $f$ tbol es reprimida y castigada, se $v$ lida en las pr cticas de los miembros de las hinchadas.

En el primer trabajo, Pablo Alabarces, en "La violencia, la academia y el fracaso", elabora un balance bibliogr fico sobre 
la violencia en el $\mathrm{f}$ tbol. De ese modo, destaca las reflexiones de los ingleses de Ian Taylor, Popplewell, Eric Dunning, Patrick Murphy, John Williams, Gary Armstrong y Richard Giulianotti. En Argentina, resalta las reflexiones pioneras de Am lcar Romero y Eduardo Archetti, continuadas con las investigaciones de Pablo Alabarces, Jos Garriga y Ver nica Moreira, desde el concepto del aguante. Adem s, repasa la intervenci $n$ estatal para enfrentar la violencia en el $f$ tbol en Inglaterra y Argentina.

En el segundo trabajo, Ver nica Moreira, en "As cualquiera tiene aguante, de fierro tiene aguante todo el mundo'. Disputas morales sobre las pr cticas violentas en el $f$ tbol", describe las transformaciones de las pr cticas y las representaciones del aguante en las hinchadas. Los cambios distinguen a los viejos miembros y a los $\mathrm{j}$ venes integrantes de la barra. Las diferencias del aguante muestran un antes, caracterizado por enfrentamientos corporales entre grupos iguales, que exclu an a hinchas comunes. $Y$ un despu s-ahora, en el que las armas de fuego son utilizadas regularmente y el ataque a los rivales puede involucrar a hinchas comunes. Por lo tanto, los $\mathrm{j}$ venes no respetan los viejos c digos del aguante.

En el tercer trabajo, Rodrigo Daskal, en "Cultura, civilizaci $n$ $y$ violencia en el $f$ tbol argentino", plantea un an lisis de la violencia, desde la categor a de cultura en los sectores populares. De ese modo, indica que las pr cticas violentas, en el entorno deportivo, exponen diferentes sentidos y valores. Los hinchas violentos, habitualmente llamados barras bravas, manifiestan una relaci $\mathrm{n}$ con el uso del cuerpo y el aguante, una categor a pr ctico-moral. El enfrentamiento corporal sirve para exhibir el aguante, manifestado en las cicatrices y en los relatos. El aguante otorga honra y prestigio, es decir, quien lo ostenta se distingue en la hinchada.

En el cuarto trabajo, Federico Czesli, en "Apuntes sobre la identidad en la hinchada de Platense", muestra que las prendas de vestir exhiben la identificaci $n$ con un equipo, se alando que 
puede ser reconocido como hincha de ese club, asumiendo las consecuencias de hacerlo visible. Por ese motivo es fundamental pelear y mantenerse en el combate. El territorio, las prendas de vestir y los tatuajes del club se deben defender mediante el enfrentamiento corporal ya que el aguante defiende el honor y la grandeza del equipo, la hinchada y sus miembros. De esa manera, se constituye la hinchada y el hincha se distingue en el grupo, al lograr la aprobaci $\mathrm{n}$ de sus integrantes.

En el quinto trabajo, Nicol s Cabrera, en "De corporalidades masculinas, aguantadoras y populares. Violencia, identidad y poder en la hinchada del Club Atl tico Belgrano", sostiene que el mando en la hinchada se organiza a partir de la posesi $n$ y la demostraci $\mathrm{n}$ del aguante. El cuerpo, la masculinidad y las pr cticas est $\mathrm{n}$ vinculados. Por eso, el hincha debe soportar $\mathrm{f}$ sicamente, las experiencias en el grupo. As es reconocido como "hombre" o "macho", opuesto a los "putos", quienes no son "verdaderos hombres", al evadir los compromisos y los h bitos. Por lo tanto, carecen de aguante.

En el sexto trabajo, Federico Fern ndez, en "Violencia y etnicidad: apuntes etnogr ficos sobre la pr ctica del $f$ tbol entre poblaciones originarias-campesinas de Jujuy (Argentina)", detaIla algunos elementos sociales, culturales e hist ricos de las poblaciones originarias y campesinas de Jujuy. Por supuesto, esas expresiones anteceden al surgimiento y la llegada del $\mathrm{f}$ tbol a la regi n. Sin embargo, esas caracter sticas se visibilizan en los lenguajes corporales, mediante la pr ctica del $f$ tbol, mostrando v nculos familiares o parentescos y estilos de juego de los habitantes de la regi $n$.

En el s ptimo trabajo, Roger Magazine y Sergio Fern ndez, en "La afici n futbol stica y la violencia en M xico: 1995-2012", detallan el proceso de trasformaci $\mathrm{n}$ de los aficionados a los equipos. En la primera etapa son grupos familiares y de amigos, en el que participan, indistintamente, hombres y mujeres. Estos grupos son llamados porras. En la segunda etapa, aparece un 
nuevo grupo, mayoritariamente masculino, que busca animar, mucho $\mathrm{m} \mathrm{s}$, al equipo. Adem s, lanzan insultos a los rivales. En la tercera etapa, surgen grupos organizados, que adoptan el t $r$ mino de "barra" y son guiados por el aguante. En consecuencia, apoyan durante todo el partido y se enfrentan, verbal y f sicamente, a los rivales. Estas "barras" se organizan en subgrupos y trasladaron sus expresiones est ticas y sus enfrentamientos a sus barrios de origen.

En el octavo trabajo, Luiz Henrique De Toledo, en "Hinchadas como pol tica en el Brasil Post-dictadura", presenta los procesos de transformaci n en las formas de "hinchar" de los seguidores de equipos de $f$ tbol. A finales de la $d$ cada del ochenta, los hinchas mostraron, nuevamente, las relaciones entre el deporte y la pol tica. La influencia del entorno electoral y los candidatos, en el contexto del $\mathrm{f}$ tbol, expusieron los $\mathrm{v}$ nculos que se pueden generar entre el juego y las elecciones. Posteriormente, el cuerpo del hincha es centro de atenci n, mediante los gestos, el exhibicionismo, los s mbolos y los enfrentamientos corporales, consecuencia de las rivalidades del $f$ tbol. Por lo tanto, las barras organizadas, torcidas, fueron responsabilizadas de la violencia en el $f$ tbol. Por ese motivo, algunos clubes promovieron programas de socio-torcedor, buscando nuevos v nculos entre los aficionados y los equipos. Asimismo, apareci la figura del hincha consumidor.

En el noveno trabajo, Alejandro Villanueva y Nelson Rodr guez, en "Aspectos legales, jur dicos y normativos sobre las barras futboleras en Bogot y Colombia", realizan un balance positivo sobre la implementaci $n$ de la pol tica $p$ blica ya que apuesta por la seguridad y la convivencia y la creaci n de comisiones locales para minimizar los riesgos en cada partido.

En el d cimo trabajo, Fernando Segura y Diego Murzi, en "Alternativas europeas comparadas de gesti $\mathrm{n}$ de la seguridad y la violencia en los estadios de $f$ tbol: tres enfoques y aplicaciones diferentes ¿Qu se puede aprender?", resaltan los aspectos 
relevantes de la legislaci $\mathrm{n}$ inglesa, belga y francesa para enfrentar y disminuir la violencia en el $f$ tbol; aspectos relacionados con la caracterizaci n de los aficionados, el rol de las fuerzas de seguridad, las condiciones ptimas de las estructuras de los estadios y las medidas que castigan las conductas violentas y tambi $n$, aquellas que estimulan el buen comportamiento. Sin embargo, en Inglaterra una de las causas que permiti disminuir la violencia en los estadios fue incrementar, considerablemente, el costo de la entrada y controlar las expresiones multitudinarias. En B Igica se han disminuido los enfrentamientos. Pero los cantos racistas y xen fobos se mantienen. $Y$ en Francia, se han aplicado algunas medidas inglesas y belgas para asumir la violencia.

En conclusi n, cada pa s debe su seguir su propio camino, seg $\mathrm{n}$ las particularidades de su violencia en el $\mathrm{f}$ tbol, tomando como gu a otros modelos.

En el und cimo trabajo, Santiago Uliana y Mat as Godio, en "Separar, dividir y mortificar. Los dispositivos culturales de seguridad en los estadios de $f$ tbol argentino", analizan el ambiente generado en los estadios y el tratamiento que ejercen las autoridades estatales sobre los hinchas. Los "operativos de seguridad" -antes, durante y despu s de los partidos- pretenden castigar el delito y las pr cticas ilegales de los hinchas, quienes pueden ser tratados como criminales o "barras bravas". Por eso, los hinchas deben ser requisados exhaustivamente, con el objetivo de localizar cualquier elemento que pueda ser usado como arma. Adem s, los grupos rivales son separados ya que cada uno es ubicado en un determinado sector.

En el duod cimo trabajo, Juan Sodo, en "De violencia a ambientes de violencia: entre el doble discurso de los hinchas y el doble reduccionismo medi tico, razones para un desplazamiento conceptual en los estudios sociales del deporte", presenta los factores que permiten el desarrollo de las pr cticas violentas en el $f$ tbol de Argentina como la historia, el organizativo-institucional, el pol tico, los jugadores, el simb lico, el paradigma de 
la seguridad, los estadios, el medi tico y el hincha com n. Los ambientes de violencia en los estadios se caracterizan por elaborar met foras criminales, responsabilizar a los integrantes de las barras de los acontecimientos violentos, suponer que se han cometido delitos y la puesta tem tica en la pantalla de la violencia en el $f$ tbol. Adem s, el hincha com $n$ solicita las pr cticas violentas, como respuesta al accionar de los rivales.

En el d cimo tercer, trabajo, Sebasti n Sustas, en "Las violencias sentenciadas. An lisis de las leyes en torno la seguridad deportiva en Argentina", explica que los estadios se han transformado en escenarios altamente custodiados por la polic a. La legislaci $\mathrm{n}$ planteada supone que, en cada partido, el peligro y la amenaza son inminentes ya que los hinchas pueden ser bastante peligrosos. De esa manera, se pretende disciplinarlos, mediante una violencia leg tima, otorgada por el Estado, se intenta disminuir la violencia en el $f$ tbol. De esa forma, conservar el orden $\mathrm{p}$ blico.

Por Itimo, en el d cimo cuarto trabajo, Jos Garriga, en "El aguante: violencias, academia y pol ticas p blicas", reitera que la violencia entre las hinchadas se denomina aguante y esa violencia es una, entre diferentes violencias en el $f$ tbol. El aguante se mide en el enfrentamiento corporal; a quien lo demuestre lo posiciona en la jerarqu a del grupo; otorga beneficios y expone un tipo de identidad en la hinchada. Por lo tanto, la violencia es una manifestaci $\mathrm{n}$ distintiva, validada y aprobada. Igualmente, el aguante no denuncia la agresi $n$ y es contrario a la legalidad.

Finalmente, el aguante y la pol tica p blica contra la violencia del $f$ tbol pueden ser propuestas de investigaci $n$ de los estudios sociales del deporte en Colombia pues permite comprender las causas de la violencia en el $f$ tbol y de esa manera, proponer una serie de medidas legislativas e institucionales incluyentes y participativas, trascendiendo as el sensacionalismo de los medios de informaci $\mathrm{n}$ y demostrar, desde los mismos gobiernos locales y nacionales, la posibilidad de prevenir los in- 
cidentes violentos entre los grupos organizados de hinchas y no continuar la ruta habitual: la represi n. Adem s, es viable promover espacios acad micos que estudien y entiendan las conductas usuales los integrantes de estas agrupaciones, investigaciones escasas en Colombia, aunque se han elaborado trabajos reflexivos, las referencias bibliogr ficas a $\mathrm{n}$ son limitadas.

Recepción 03-06-2015

Aprobación: 11-12-2015 